\title{
The evolution of big ideas
}

\author{
Molecular biology has seen many grand visions come and go and some others come back
}

\author{
Philip Hunter
}

$\mathrm{T}$ he history of science is littered with big ideas-from the Earth being a globe, to the theory of gravity, to phlogiston theory, to the theory of evolution and cold fusion-some of which eventually ended up on the great heap of disproven theories as new knowledge proved them to be wrong or unfeasible. In biomedical research, grand visions include the Human Genome Project (HGP) or the Human Brain Project, therapeutic approaches such as gene therapy or regenerative medicine, or radically new methodologies like genomics or systems biology. All of these ideas have generated much excitement if not hype mainly about their potential to cure diseases or improve human health. Some of these ideas eventually delivered on their promises, whereas many other putative breakthroughs have faded from public attention.

\section{"The tendency to get excited by new ideas is intrinsic to the human condition and should be embraced..."}

The tendency to get excited by new ideas is intrinsic to the human condition and should be embraced even if our demand for success and affirmation tends to greatly exceed the rate at which scientific research can deliver results, commented Anthony Nicholls, President and CEO of OpenEye Scientific Software, a privately held company based in Santa Fe, USA, which develops large-scale molecular modelling applications and toolkits. "The basic thesis is that when working in an endeavour where success is rare, people tend to over react to apparent success by what I call a 'dopamine reinforcement’ loop”, he said. "Expectation of success raises dopamine levels which makes one less discerning, which increases the expectations of success on more limited data and so on. I think we all do it, especially when we are imaginative and can make good stories about why something 'ought' to work".

\section{Counter reaction}

However, the counter reaction to the initial excitement or hype can be more damaging than the hype itself if people lose faith or if funding dries up. "Sometimes the danger here is that there is too dramatic a shift of the pendulum after the first claims have turned out to be exaggerated", commented Harold Varmus, who was Director of the US National Cancer Institute until March 2015 before he returned to his native New York as Professor of Medicine at Weill Cornell Medicine and Senior Associate at the New York Genome Centre. He gave two examples of fields - the early genome projects and eventually gene therapy - that have survived the early deflation following exaggerated claims. The spate of genome projects, especially the HGP in the 1990s, generated many claims of revolutionary cures, and it still suffers to an extent from this negative perception among the media and public today. "I hear people say we've had the human genome for the last 15 years and still there's lots of disease", said Varmus, who won the 1989 Nobel Prize in Physiology and Medicine. But that, he argued, misrepresented both the objectives and achievements of the HGP. "Everyone I know who does serious work uses the genome every day, whether mouse, worm or human genome. It was one of those events up there with discovery of the genetic code".

Varmus commented that in some fields the hype got so far ahead of reality that it took a long time to recover from the subsequent negative reaction, which diminished funding and discouraged young scientists from entering. "I do think there are places where excessive claims have been detrimental to the exercise, gene therapy being one. Certainly in the 1990s it was clear the confidence people had in gene therapy was out of proportion to what was happening or feasible", he said. This confidence was built on a cure of severe combined immune deficiency in the late 1990s, which was developed by Alain Fisher at the Hospital Necker in Paris, France. Yet, since this early breakthrough, gene therapy stalled for more than two decades until recently when an international team of researchers under the guidance of the UK Cystic Fibrosis Gene Therapy consortium succeeded in introducing a normal copy of a gene located on chromosome 7 the mutation of which causes cystic fibrosis. This mutation was first identified in 1989 [1], but it took more than 25 years-owing to the difficulty of safely delivering the corrective gene-before the first successful phase III trial in 2015 [2]. The trial may be a proof of concept rather than a long-term solution, but it shows progress towards an effective gene therapy for cystic fibrosis.

(c)

"... in some fields the hype got so far ahead of reality that it took a long time to recover from the subsequent negative reaction"

\section{Dashed hopes}

Whereas gene therapy is slowly picking up speed again, similar hopes about 
molecular-targeted cancer therapy were eventually dashed, at least temporarily. "This mode of therapy has been heavily promoted, where a particular mutant or over-expressed signalling protein, almost always a protein kinase, is targeted with a highly specific pharmacologic inhibitor", explained Robert Weinberg, Director of the MIT Ludwig Centre for Molecular Oncology in Massachusetts, USA. He commented that it was an elegant achievement in molecular biology and organic chemical synthesis, but still proved to be a false dawn. "It has the result that a certain proportion of patients, often less than a quarter of all cases, respond for a half year and then relapse, their tumours having developed resistance to the targeted drug", he explained. "Many, myself included, were deluded into thinking that the case of chronic myelogenous leukaemia and the truly brilliant success of Gleevec/imatinib would represent the 'poster child' of a new era of anti-cancer therapy where the notion that such success would prove generalizable to many other types of cancer, which has not proven to be the case sadly".

\section{"In particular, biomedical research that is linked to disease risks falling victim to early hopes and hype and goes through the trough of disillusionment that follows"}

First approved in the USA in 2001, imatinib, a tyrosine-kinase inhibitor against multiple cancers, achieved significant successes, but the ability of cells to develop mutations means that tumour cells develop resistance over time, leading to relapse in some cases [3]. This was not so much hype, but more a case of hope triumphing over past experience that when new treatments look too good to be true, they usually are, often because the underlying complexities of the molecular biology have been overlooked. It does not invalidate the idea of molecular targeting, but is a reminder of the need for caution in declaring a therapy revolutionary before it has stood the test of time.

Another potential ground-breaking cancer therapy involves targeting apoptosis, the natural process of programmed cell death by which multicellular organisms rid themselves of unwanted cells. As this includes tumour cells, cancer can only progress by blocking apoptotic mechanisms. Thus, these inhibitory processes are obvious therapeutic targets, but progress has been slower than had been anticipated. "Apoptosis lies at the heart of cancer biology and is absolutely an interesting concept in therapy", said Jarle Breivik, a specialist in the evolutionary basis of cancer at the University of Oslo in Norway. "But it also illustrates why we cannot treat ourselves out of the cancer epidemic. Our cells are genetically programmed to self-destruct in response to DNA damage and other signs of ageing. These apoptotic mechanisms prevent the cells from developing into cancer. Concurrently, cells are living entities subject to the principle of evolution, and those cells that avoid apoptosis will have a selective advantage relative to neighbouring cells that succumb to apoptosis. In essence, our cells have two alternative endgames: they can die due to ageing and apoptosis or they can survive as cancers. Either way, the organism is doomed, and the relationship between cancer and apoptosis demonstrates the fact that we are not designed to live forever".

Again, it is the complexity of the underlying biology that has stalled developing cures based on apoptosis after the initial excitement. "Inducing apoptosis is the endgoal of many, many anti-cancer therapies", Weinberg explained. "However, doing so by interfering directly with the apoptosisregulating machinery has proven more challenging than one had hoped, given the extraordinary complexity of pro-apoptotic and countervailing anti-apoptotic regulators. The molecular biology has proven to be more complex and subtle than many had anticipated".

In particular, biomedical research that is linked to disease risks falling victim to early hopes and hype and goes through the trough of disillusionment that follows. In the case of the microbiome, the jury is still out, according to Arturo Casadevall, an immunologist and head of laboratory at the Albert Einstein School of Medicine in Baltimore, USA. The hope is that manipulation of the human microbiome, especially bacteria in the gut, can inhibit some infectious diseases or treat metabolic conditions such as Crohn's disease and type 2 diabetes. "The microbiome area is definitively in danger of being overhyped", Casadevall said. "Many groups are making correlations between the microbiome and specific conditions without being able to establish causality”. He is still confident though that it will eventually yield therapeutic approaches, but it would require clearer results that can be replicated. "There is no question in my mind that we are in the midst of a microbiome revolution with regards to learning about our microbes but many of the associations being made are weak", Casadevall noted.

\section{General trends and methodologies}

Exaggeration and inflated expectations do not only befall individual fields of research or projects such as apoptosis or the HGP, but can also affect general trends in research and medicine. This is true of personalized medicine, according to Neil Greenspan, an immunologist at the Case Western Reserve University School of Medicine in Cleveland, USA. "Claims for personalized medicine are sometimes realized, but more frequently, genotypic information is not sufficient to precisely determine truly personal risk or therapy”, he said.

\section{"Exaggeration and inflated expectations do not only befall individual fields of research or projects [...] but can also affect general trends in research and medicine"}

The same applies to a number of general themes or methodologies, which are not focused on a therapeutic endgame, but promise to deepen our understanding of fundamental biology. This is, for instance, the case for Big Data and Deep Learning, which have been imported from Information Technology. These technologies have been valuable in some applications, such as customer recommendations in retail or image recognition. But these successes have not been replicated in biology, Nicholls argued. "Interestingly, Deep Learning consistently does a little better than the methods which have proceeded it, from what I have seen of a number of studies, but that difference is unimportant in the real world-quite unlike its success in domains such as image recognition", he 
said. Nicholls is similarly sceptical about the value of systems biology as it has yet to deliver any significant advances after 15 years. Varmus, however, disagrees, contending that systems approaches have already helped to answer some fundamental questions. One example is analysing pathways of fatty acid oxidation in microorganisms with potential for the biosynthesis of fuels and fine chemicals [4].

Synthetic biology, the applied side of systems biology, which has also been accused of being overhyped, is now maturing into a serious field of biotechnology delivering useful applications, according to Luis Serrano, Director of the CRG-Centre for Genomic Regulation in Barcelona, Spain. The field started with manipulating bacteria using recombinant DNA technology but has developed to embrace much broader designs via standardized rational methods. Serrano, along with others in the field, argues that the methods and tools to efficiently design synthetic regulatory and metabolic pathways are now available to develop valuable products of societal value. Yet, Serrano noted that it still requires a significant amount of trial and error because it is not possible to predict exactly the behaviour of an engineered bacterium. Varmus though argues that synthetic biology is a misnomer in that it has not actually achieved what it set out to do. "My complaint is that it has not created life and is not different in principle from what has been done in molecular biology for years. It implies you are making life and yet nobody has redesigned how organisms work", he said.

\section{The value of knowledge itself}

The latest hot topic that is currently attracting enormous attention and hype is the CRISPR/ Cas technology and its potential use to cure human diseases. Originally discovered in bacteria as part of an innate immune system against phage infections, scientists developed it into a highly efficient tool to edit genes. CRISPR/Cas is not only cheap and easy to use, but it is also highly specific and programmable in terms of its target sequence, which makes it an ideal tool for a wide range of potential applications in agriculture, medicine, basic research and synthetic biology. In particular, the prospect of being able to fix deleterious or disease-causing mutations in humans has triggered not just great hopes but also intense debates about the ethics of editing human embryos and the human germline. However, as Casadevall noted, the jury is still out on the impact of CRISPR for human disease. "We are all hopeful and there is a lot of hype for curing genetic diseases but we still have to see if the technology can be made to work safely", he said.

(c) ... even fields that have not yet yielded new cures, [...] have nonetheless greatly expanded knowledge often in unexpected ways"

Yet, most hype, promises and hopes mainly concern the application of new knowledge to improve the human condition, in particular in medicine. However, even fields that have not yet yielded new cures, such as apoptosis or research on the microbiome, have nonetheless greatly expanded knowledge often in unexpected ways. Irrespective of any practical expectations, this is a point that we should not lose sight of, according to Varmus. "The genome work of the 1990s has proved even more important than I thought it would be", he said. "Understanding who we are as human beings has been dramatically transformed by the human genome. Who would have thought we would have got the Neanderthal Genome by now?"

\section{References}

1. Rommens JM, Iannuzzi MC, Kerem B, Drumm ML, Melmer G, Dean M, Rozmahel R, Cole JL, Kennedy D, Hidaka N et al (1989) Identification of the cystic fibrosis gene: chromosome walking and jumping. Science 245: 1059-1065

2. Alton EW, Armstrong DK, Ashby D, Bayfield KJ, Bilton D, Bloomfield EV, Boyd AC, Brand J, Buchan R, Calcedo R et al (2015) Repeated nebulisation of non-viral CFTR gene therapy in patients with cystic fibrosis: a randomised, double-blind, placebo-controlled, phase $2 \mathrm{~b}$ trial. Lancet Respir Med 3: 684-691

3. Gambacorti-Passerini CB, Gunby RH, Piazza

R, Galietta A, Rostagno R, Scapozza L (2003)

Molecular mechanisms of resistance to imatinib in Philadelphia-chromosomepositive leukaemias. Lancet Oncol 4: $75-85$

4. Cintolesi A, Rodríguez-Moyá M, Gonzalez R (2013) Fatty acid oxidations: systems analysis and applications. Wiley Interdiscip Reu Syst Biol Med 5: 575-585 